that a negative gate voltage substantially reduces the angular spread of electrons emerging from the point contact. The use of gates as energy selectors (or spectrometers) has been demonstrated experimentally; in one case, gates were used to make a ballistic, hot-electron transistor with considerable gain that operates at liquid-helium temperature ${ }^{5}$. In the proposed focusing transistor, after electrons emerge from a point contact, the gate restricts which of them travel into the detector on the right. The optimum focusing of the gate occurs when the electrons draw up a potential barrier that exceeds their energy, so that they are transported across the device by tunnelling through the barrier.

The analysis of the proposed tunnelling transistor ignores one salient feature of the physics: the authors assume that the walls of the point contact are perfectly square. In practice, the corners of any real device will be rounded somewhat (either

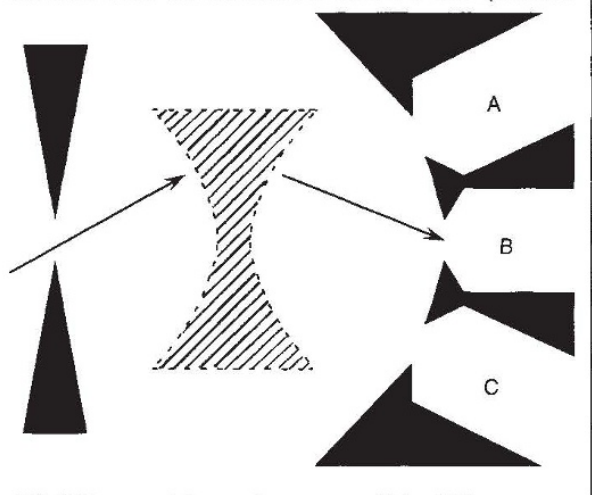

Injector Lens gate Detectors

FIG. 3 The electron-lens transistor ${ }^{4}$ works in simple analogy with classical optics to focus electrons from the point contact on the left into a particular contact (B) on the right.

because of imperfect lithography or because the electrons themselves will screen the large electric fields at the corners), so that the incoming electrons tend to see barriers with round corners. This rounding leads to an adiabatic coupling of the transport modes in the narrow region to those in the injector ${ }^{6}$ and to an extra collimation of the incoming beam ${ }^{7.8}$, so that even in the absence of the tunnelling gate, the outgoing beam tends to lie along the axis of the device. This collimation was recently confirmed in an experiment by Molenkamp et al. ${ }^{9}$. The proposed transistor also has another drawback. To be of practical use, it must be able to carry a substantial current, but the use of tunnelling to focus the electrons places severe limits on the throughput. Most of the input current is reflected back into the injector by the gate.

An ingenious approach to focusing without severely limiting the current has been devised by Sivan, Heiblum, Umbach and Shtrikman ${ }^{4}$, who use a classical lens to focus the electrons injected through a point contact as depicted in Fig. 3. This method does not require tunnelling through the lens, but relies instead on simply changing the electron wavelength in the region under the gate. This refracts the beam of electrons just as a bit of curved glass refracts a beam of light. In the case of electrons, the focusing lens is convex and not concave as is the lens for light. The essential reason for the difference is that electrons in the gated region have lower energy and therefore longer wavelength, whereas the energy (and therefore the frequency) of the photons is fixed so that moving more slowly in the denser medium, they have shorter wavelength. The result is that whereas a convex lens is required to focus a columnar beam of light to a point, to focus the electron beam, one must use the opposite curvature - a concave lens. One can also apply a perpendicular magnetic field to such a transistor to guide the beam into one of the other contacts. For instance, a small field out of the page will push the focused beam into contact $A$, whereas reversing the field would focus the beam at $\mathrm{C}$.

The practical usefulness of any of these devices is still very much in doubt. For logic circuits, the output of one transistor must be able to drive the gate of the next (and preferably several at once). The voltage scales in the focusing devices are off by orders of magnitude from achieving this goal. Typical gate voltages are $100 \mathrm{mV}$, but typical output voltages ${ }^{4,10}$ are a few microvolts. To conquer this disparity, the transistors would have to operate under very extreme conditions: extremely low carrier density, for instance. Under such stringent conditions, the fraction of such critically adjusted devices that actually work will be very small. Even if there were a successful yield, then the very small width reduces again the available current flow. In short, because of these and other technical problems" near future, these transistors can be made to perform useful functions as amplifiers or logic switches.

S. Washburn is at the IBM T. J. Watson Research Center, Yorktown Heights, New York 10598, USA.

1. van Houten, H. et al. Phys. Rev. B39, 8556-8575 (1989).

2. de Raedt, H., Garcia, N. \& Sáenz, J.J. Phys. Rev. Lett. 63. 2260-2263 (1989).

3. Heiblum, M. \& Fischetti, M.V. in Topics in Current Physics (ed. Capasso, F.) (Springer, Berlin, in the press)

4. Sivan, U., Heiblum, M., Umbach, C.P. \& Shtrikman, H. Phys. Rev. Lett. (submitted)

5. Palevski, A., Umbach, C.P. \& Heiblum, M. Appl. Phys. Lett. 55, 1421-1423 (1989)

6. Glazman, L.I., Lesovik, G.B., Khmeltnisky, D.E. \& Schecter, R.J. JETP Lett. 48, 238--241 (1988)

7. Beenakker, C.W.J. \& van Houten, H. Phys. Rev. B39 10445-10448 (1989).

8. Baranger, H.U. \& Stone, A.D. Phys. Rev. Lett. 63 414-417 (1989)

9. Molenkamp, L.W. et al. Phys. Rev. B41, 1247 (1990).

10. Spector, J.S. et al. Pap. presented at Symp. New Phenom. Mesoscopic Structures (Kona, Hawaii, 1989). 11. Landauer, R. Phys. Today 42 No. 10, 119-121 (1989).

\section{Life in reverse}

THE basic molecules of life all have a specific handedness. Its amino acids are all left-handed, whereas its sugars are righthanded. Molecules of opposite handedness can be synthesized, but are biochemically useless, or even poisonous.

The very first life forms may well have had all sorts of biochemical chiralities; but the ensuing 'war' between them was decisively won by the present arrangement. A biochemically 'inverted' animal, if one could be created, would be quite unable to digest any food it could find in our world. An inverted plant, however, is quite feasible. Plants build themselves entirely from small molecules like water and carbon dioxide, which have no handedness. So Daedalus is now trying to create the world's first inverted plant.

His starting point is a minute exception to the general rule. The micro-organism Bacillus licheniformis weaves its cell wall from right-handed amino acids in an effort to make itself indigestible to phagocytes. DREADCO's biochemists are intrigued: surely it cannot have mirror-image DNA? More likely some small 'switch' in DNA determines the handedness of its products, and $B$. licheniformis has ingeniously set this switch oppositely to everyone else. The DREADCO team hopes to extract the switch by standard genetic engineering methods, and to insert it into various promising plants.

The most promising, from Daedalus's point of view, are the structural and textile plants like oak, pine, flax, cotton and so on. They would be immune to wood borers and boll weevils; and would yield timber, paper and textiles which would magically resist rot and decay. Inverted timber would revolutionize the construction industry, which in rain-soaked Britain is paranoid about rot and fungal attack. Inverted wallpaper would never go mouldy, and inverted clothing would be moth proof.

Another DREADCO goal is inverted vegetables. They may taste a bit strange (some chiral molecules taste differently to their mirror images), but with luck they will be harmless and quite acceptable to the human palate. To the human stomach, however, they will be untouchable and totally devoid of nourishment. Inverted lentils, nuts and brown rice, combining vegetarian rectitude with zero delivered calories, would be an ideal slimming diet for earnest macrobiotic folk.

A stand or field of the new plants will rapidly get cluttered up with decay-proof stalks, leaves, fallen timber, and so on. Perhaps $B$. licheniformis might mutate to exploit this residue, starting a closed local 'inverted ecology'. Its competition with the surrounding normal ecology might mimic the primaeval war of the chiralities most instructively.

David Jones 